\title{
A sublinear variance bound for solutions of a random Hamilton-Jacobi equation
}

\author{
Ivan Matic* and James Nolen ${ }^{\dagger}$
}

\begin{abstract}
We estimate the variance of the value function for a random optimal control problem. The value function is the solution $w^{\epsilon}$ of a Hamilton-Jacobi equation with random Hamiltonian $H(p, x, \omega)=$ $K(p)-V(x / \epsilon, \omega)$ in dimension $d \geq 2$. It is known that homogenization occurs as $\epsilon \rightarrow 0$, but little is known about the statistical fluctuations of $w^{\epsilon}$. Our main result shows that the variance of the solution $w^{\epsilon}$ is bounded by $O(\epsilon /|\log \epsilon|)$. The proof relies on a modified Poincaré inequality of Talagrand.
\end{abstract}

\section{Introduction}

In this paper we study the random optimal control problem

$$
u(t, x, \omega)=\sup _{\gamma \in \mathcal{A}_{t, x}} g(\gamma(t))-\mathcal{L}(\gamma, \omega), \quad x \in \mathbb{R}^{d}, \quad t>0
$$

in dimension $d \geq 2$, where the supremum is taken over the set of admissible paths

$$
\mathcal{A}_{t, x}=\left\{\gamma \in W^{1, \infty}\left([0, t] ; \mathbb{R}^{d}\right) \mid \gamma(0)=x\right\}
$$

The upper-semicontinuous payoff function $g: \mathbb{R}^{d} \rightarrow \mathbb{R} \cup\{-\infty\}$ is given. The cost functional $\mathcal{L}$ has the form

$$
\mathcal{L}(\gamma, \omega)=\int_{0}^{t} L\left(\gamma^{\prime}(s), \gamma(s), \omega\right) d s=\int_{0}^{t} K\left(\gamma^{\prime}(s)\right)+V(\gamma(s), \omega) d s
$$

where $K(p): \mathbb{R}^{d} \rightarrow[0, \infty)$ is convex and grows super-linearly in $|p|$. The function $V(x, \omega)$ is a scalar random field that is statistically stationary and ergodic with respect to certain translations in $x$. The parameter $\omega \in \Omega$ denotes a sample from a given probability space $(\Omega, \mathcal{F}, \mathbb{P})$. Thus, the value function $u(t, x, \omega)$ is random. Our main result shows that the variance of $u(x, t, \omega)$ grows only sublinearly in $t$ as $t \rightarrow \infty$.

Under certain conditions on $g$ and $L, u(t, x, \omega)$ is uniformly continuous and is a viscosity solution [3] of the random Hamilton-Jacobi equation

$$
\left\{\begin{array}{l}
u_{t}=H(D u, x, \omega), \quad x \in \mathbb{R}^{d}, \quad t>0 \\
u(0, x)=g(x), \quad x \in \mathbb{R}^{d},
\end{array}\right.
$$

\footnotetext{
*Mathematics Department, Duke University, Box 90320, Durham, North Carolina, 27708, USA. (matic@math.duke.edu).

${ }^{\dagger}$ Mathematics Department, Duke University, Box 90320, Durham, North Carolina, 27708, USA. (nolen@math.duke.edu).
} 
where $H(p, x)=K^{*}(p)-V(x), K^{*}$ being the Legendre transform of $K$. For simplicity, consider the case where $g(x)=\eta \cdot x$ is a linear function. Then for each $\epsilon>0$, the function $w^{\epsilon}(t, x, \omega)=$ $\epsilon u(t / \epsilon, x / \epsilon, \omega)$ solves the initial value problem

$$
\left\{\begin{array}{l}
w_{t}^{\epsilon}=H\left(D w^{\epsilon}, \frac{x}{\epsilon}, \omega\right), \quad x \in \mathbb{R}^{d}, \quad t>0 \\
w^{\epsilon}(0, x)=\eta \cdot x=g(x) .
\end{array}\right.
$$

For certain Hamiltonians $H(p, x, \omega)$ which are convex in $p$, statistically stationary and ergodic with respect to translation in $x$, it is known [11, 13, that as $\epsilon \rightarrow 0$, homogenization occurs (see also [2, 9] for alternative proofs and [6, 7, 8, 12] for related results). This means that the functions $w^{\epsilon}(t, x, \omega)$ converge locally uniformly in $[0, \infty) \times \mathbb{R}^{d}$, as $\epsilon \rightarrow 0$ to the deterministic function $\bar{w}(t, x)$ which solves

$$
\left\{\begin{array}{l}
\bar{w}_{t}=\bar{H}(D \bar{w}), \quad x \in \mathbb{R}^{d}, \quad t>0 \\
\bar{w}(0, x)=g(x) .
\end{array}\right.
$$

The function $\bar{H}(p): \mathbb{R}^{d} \rightarrow \mathbb{R}$ is called the effective Hamiltonian. We may think of this convergence as kind of law of large numbers for $w^{\epsilon}$, although the limit $\bar{w}$ and the effective Hamiltonian $\bar{H}$ are not determined by a simple averaging. Beyond this convergence result, relatively little is known about the properties of $\bar{H}$, about the rate of convergence $w^{\epsilon} \rightarrow \bar{w}$, or about the statistical behavior of $w^{\epsilon}-\mathbb{E}\left[w^{\epsilon}\right]$, where $\mathbb{E}[\cdot]$ denotes expectation with respect to the probability measure $\mathbb{P}$. Our work pertains to this last issue: in terms of $w^{\epsilon}(t, x, \omega)$, our estimate on the variance of $u$ implies that $\operatorname{var}\left(w^{\epsilon}(t, x, \omega)\right) \leq C \epsilon /|\log \epsilon|$, as $\epsilon \rightarrow 0$.

Before stating our main result, let us make some definitions and assumptions more precise. We will suppose the random field $V(x, \omega)$ has the following special structure. Let $a<b$ be two real numbers. Let $\Omega=\{a, b\}^{\mathbb{Z}^{d}}$ be the set of all functions $\omega: \mathbb{Z}^{d} \rightarrow\{a, b\}$. Let the probability measure $\mathbb{P}$ be the shift-invariant product measure on $\Omega$ determined by $\mathbb{P}\left(\omega_{k}=a\right)=\alpha$ and $\mathbb{P}\left(\omega_{k}=b\right)=\beta$, for all $k \in \mathbb{Z}^{d}$, where $\alpha \in(0,1)$ and $\beta=1-\alpha$. Thus the random variables $\left\{\omega_{k}\right\}_{k \in \mathbb{Z}^{d}}$ are independent and identically distributed. Now for $k \in \mathbb{Z}^{d}$, let $Q_{k}=k+[0,1)^{d}$ denote the unit cube with corner at the point $k$. Given $\omega \in \Omega$, define $V(x, \omega): \mathbb{R}^{d} \times \Omega \rightarrow\{a, b\}$ by

$$
V(x, \omega)=\sum_{k \in \mathbb{Z}^{d}} \omega_{k} \mathbb{I}_{Q_{k}}(x),
$$

with $\mathbb{I}_{Q_{k}}$ is the indicator function for the set $Q_{k}$. Thus, $x \mapsto V$ is piecewise constant, taking values $a$ or $b$ on the unit cubes. By construction, the law of $V(x, \omega)$ is the same as that of $V(x+k, \omega)$ for any $k \in \mathbb{Z}^{d}$. This precise construction of the field $V(x, \omega)$ is not essential for our result to hold. In particular, the function could be mollified so that it is uniformly continuous, or $V(x, \omega)$ could depend on the values of $\omega_{k}$ for $k$ in a bounded neighborhood of $x$. Nevertheless, the choice of $\mathbb{P}$ as the product measure on $\Omega=\{a, b\}^{\mathbb{Z}^{d}}$ is motivated by the main analytical tool presented below in Theorem 1.2 .

We suppose that $K: \mathbb{R}^{d} \rightarrow[0, \infty)$ is convex, $K(0)=0$, and that

$$
\lim _{|z| \rightarrow \infty} \frac{K(z)}{|z|}=+\infty
$$

For the case of dimension $d=2$ we will make use of an extra non-degeneracy condition: for some $\nu>1$,

$$
K(z) \geq|z|^{\nu} \quad \forall z \in B_{1 / 2}(0) .
$$

Given $V$ and $K$, let $L(p, x, \omega)=K(x)+V(x, \omega)$ and let $u$ be defined by (1.1). The following estimate of the variance of $u$ for large $t$ is our main result: 
Theorem 1.1 Let $d \geq 2$. Let $x \in \mathbb{R}^{d}$ and suppose that $g: \mathbb{R}^{d} \rightarrow \mathbb{R} \cup\{-\infty\}$ satisfies

$$
g(y)<g(x)+C_{1}(1+|y-x|), \quad \forall y \in \mathbb{R}^{d} .
$$

There is a constant $C>0$, depending only on $C_{1}, K, \alpha, \beta$, and $|b-a|$, such that

$$
\operatorname{var}(u(t, x, \omega)) \leq C \frac{t}{\log t}, \quad \forall t \geq 2 .
$$

The main tool that we use to control the variance of $u(t, x, \omega)$, is the following theorem, which is a slight variation of an inequality of Talagrand (see [14, Theorem 1.5). This result holds for product spaces of the form $\Omega_{J}=\{a, b\}^{J}, J$ being a finite set, and $\mathbb{P}$ being the product measure on $\Omega_{J}$ with marginals $\mathbb{P}\left(\omega_{j}=a\right)=\alpha \in(0,1)$ and $\mathbb{P}\left(\omega_{j}=b\right)=\beta=1-\alpha$, for all $j \in J$. Let us define $\phi_{j} \omega$ to be the element of $\{a, b\}^{J}$ which is identical to $\omega$ except that the $j$-th component $\omega_{j}$ is opposite to $\omega_{j}$. That is, $\phi_{j} \omega=\omega^{\prime}$, where $\omega_{k}^{\prime}=\omega_{k}$ for $k \neq j$, and $\omega_{j}^{\prime} \neq \omega_{j}$. For each random variable $f: \Omega_{J} \rightarrow \mathbb{R}$ define $\sigma_{j} f(\omega)=f\left(\phi_{j} \omega\right)$ and

$$
\rho_{j} f(\omega)=\frac{\sigma_{j} f(\omega)-f(\omega)}{2} .
$$

Theorem 1.2 There is a constant $C>0$, independent of $|J|$, such that

$$
\operatorname{var}(f) \leq C \sum_{j \in J} \frac{\left\|\rho_{j} f\right\|_{2}^{2}}{1+\log \frac{\left\|\rho_{j} f\right\|_{2}}{\left\|\rho_{j} f\right\|_{1}}}
$$

holds for all $f \in L^{2}\left(\Omega_{J}\right)$.

The idea of using this inequality to estimate the variance of $f(\omega)=u(t, x, \omega)$ comes from the work of Benjamini, Kalai, and Schramm [4] who used this inequality to estimate the distance variance in first passage percolation, a problem which has some features similar to the control problem (1.1). Specifically, they consider the length of minimal paths between two points in the integer lattice $\mathbb{Z}^{d}$ under a random metric. Each edge $e$ in the nearest-neighbor graph is assigned an independent random weight $\omega_{e} \in\{a, b\}$, and the length of a path between two points $x, y \in \mathbb{Z}^{d}$ is defined as the sum of the edge weights along a path connecting $x$ and $y$. They proved that $\operatorname{var}\left(d_{\omega}(0, v)\right) \leq$ $C|v| / \log |v|$, where $d_{\omega}(0, v)$ is the length of the shortest path connecting 0 and $v$. See [5] for some extensions of that result. The main difficulty in applying the ideas of [4] to the present setting comes from the different structure of the cost functional $\mathcal{L}(\gamma, \omega)$, which necessitates more control on the optimizing paths.

As we have mentioned, for $d \geq 2$ there are relatively few results about the random fluctuation of $u(t, x, \omega)$ (as $t \rightarrow \infty)$ or $w^{\epsilon}(t, x, \omega)$ (as $\epsilon \rightarrow 0$ ). In [10], Rezakhanlou derived conditions under which a central limit theorem holds for $w^{\epsilon}(t, x)$ where $w^{\epsilon}$ is the solution of the Hamilton-Jacobi equation (1.3), i.e. whether $\epsilon^{-1 / 2}\left(w^{\epsilon}-\bar{w}\right)$ converges in law to some nontrivial stochastic process as $\epsilon \rightarrow 0$. In the case $d=1$ those conditions can be verified for Hamiltonians having the form $H(p, x, \omega)=K(p)-V(x, \omega)$, and the limit distribution can be computed (see Corollary 2.6 in [10]). For $d \geq 2$, however, it is difficult to verify those conditions. Indeed, our result shows that we may have $\operatorname{var}\left(w^{\epsilon}\right)=o(\epsilon)$, which is less than what a CLT as in [10] would suggest. As this paper was being written, we learned of another work by Armstrong, Cardaliaguet, and Souganidis [1], who study the rate of convergence $w^{\epsilon} \rightarrow \bar{w}$. Our Theorem 1.1 pertains to the variance of $w^{\epsilon}$, i.e the statistical error $w^{\epsilon}-\mathbb{E}\left[w^{\epsilon}\right]$, but does not give an estimate of the bias $\mathbb{E}\left[w^{\epsilon}\right]-\bar{w}$.

The paper is organized as follows. In Section 2 we derive some properties of the paths $\gamma$ which nearly optimize (1.1). Section 3 contains the main argument for the proof of Theorem 1.1. Section 4 and Section 5 contain proofs so some technical estimates needed in Section 3 . 
Acknowledgement The work of JN was partially funded by grant DMS-1007572 from the US National Science Foundation.

\section{Properties of optimizing paths}

Without loss of generality, let us suppose $x=0$ and simply write $u(t, \omega)=u(t, 0, \omega)$ and

$$
\mathcal{A}_{t}=\mathcal{A}_{t, 0}=\left\{\gamma \in W^{1, \infty}\left([0, t] ; \mathbb{R}^{d}\right) \mid \gamma(0)=0\right\} .
$$

So, we are studying the quantity

$$
u(t, \omega)=\sup _{\gamma \in \mathcal{A}_{t}} g(\gamma(t))-\mathcal{L}(\gamma, \omega) .
$$

for some function $g: \mathbb{R}^{d} \rightarrow \mathbb{R} \cup\{-\infty\}$, with $g(0) \in \mathbb{R}$. For $\delta>0$ and $\omega \in \Omega$, let $M_{\delta}(t, \omega)$ be the set of all paths $\gamma \in \mathcal{A}_{t}$ such that

$$
g(\gamma(t))-\mathcal{L}(\gamma, \omega) \geq u(t, \omega)-\delta .
$$

For each $\delta>0$, this set is non-empty, and we refer to these paths as $\delta$-approximate optimizers. Observe that $M_{\delta_{2}}(t, \omega) \subset M_{\delta_{1}}(t, \omega)$ if $0<\delta_{2}<\delta_{1}$. If an optimal path $\gamma$ exists, meaning that $u(t, \omega)=g(\gamma(t))-\mathcal{L}(\gamma, \omega)$, then it is certainly an approximate optimizer for any $\delta>0$. In this section we derive some useful properties of approximate optimizers which will be used in the proof of Theorem 1.1.

\section{Deterministic Bounds}

First, we have a few estimates which do not involve the random structure of the control problem.

Lemma 2.1 Let $\gamma \in M_{\delta}(t, \omega)$. Then for any $r_{1}, r_{2} \in[0, t]$,

$$
\int_{r_{1}}^{r_{2}} L\left(\gamma^{\prime}(s), \gamma(s), \omega\right) d s \leq\left(r_{2}-r_{1}\right) b+\left(r_{2}-r_{1}\right) K\left(\frac{\gamma\left(r_{2}\right)-\gamma\left(r_{1}\right)}{r_{2}-r_{1}}\right)+\delta
$$

and

$$
\int_{r_{1}}^{r_{2}} L\left(\gamma^{\prime}(s), \gamma(s), \omega\right) d s \geq\left(r_{2}-r_{1}\right) a+\left(r_{2}-r_{1}\right) K\left(\frac{\gamma\left(r_{2}\right)-\gamma\left(r_{1}\right)}{r_{2}-r_{1}}\right)
$$

Proof of Lemma 2.1: Given $\gamma \in M_{\delta}(t, \omega)$, define a new path $\hat{\gamma} \in \mathcal{A}_{t}$ according to

$$
\hat{\gamma}(s)=\gamma\left(r_{1}\right)+\left(s-r_{1}\right) \frac{\gamma\left(r_{2}\right)-\gamma\left(r_{1}\right)}{r_{2}-r_{1}} s \in\left[r_{1}, r_{2}\right]
$$

and $\hat{\gamma}(s)=\gamma(s)$ for $s \notin\left[r_{1}, r_{2}\right]$. Thus we have replaced a section of $\gamma$ with a straight-line path connecting the same points. Since $\gamma \in \mathcal{M}_{\delta}(\omega)$, we must have $\mathcal{L}(\gamma, \omega) \leq \mathcal{L}(\hat{\gamma}, \omega)+\delta$. In particular,

$$
\begin{aligned}
\int_{r_{1}}^{r_{2}} L\left(\gamma^{\prime}, \gamma, \omega\right) d s & \leq \int_{r_{1}}^{r_{2}} L\left(\hat{\gamma}^{\prime}, \gamma, \omega\right) d s+\delta \\
& \leq\left(r_{2}-r_{1}\right) b+\left(r_{2}-r_{1}\right) K\left(\frac{\gamma\left(r_{2}\right)-\gamma\left(r_{1}\right)}{r_{2}-r_{1}}\right) .
\end{aligned}
$$

This proves (2.11). The lower bound (2.12) follows from Jensen's inequality, the convexity of $K$, and the fact that $V(x, \omega) \geq a$. 
Lemma 2.2 Suppose that $g: \mathbb{R}^{d} \rightarrow \mathbb{R} \cup\{-\infty\}, g(0) \in \mathbb{R}$, and

$$
g(y)<g(0)+C_{1}(1+|y|), \quad \forall y \in \mathbb{R}^{d} .
$$

There is a constant $R$ depending only on $K, C_{1}$, and $b-a$ such that

$$
\left|\gamma\left(t_{2}\right)-\gamma\left(t_{1}\right)\right| \leq R\left(1+\left|t_{1}-t_{2}\right|\right), \quad \forall t_{2}, t_{1} \in[0, t]
$$

holds for all paths $\gamma \in M_{1}(t, \omega)$ and all $t>1$.

Proof of Lemma 2.2; We first show there is a constant $R_{0}$ depending only on $K, C_{1}$, and $b-a$ such that

$$
|\gamma(t)-\gamma(0)| \leq t R_{0}
$$

holds for all $\gamma \in M_{1}(t, \omega)$ and all $t \geq 1$. Define the path $\hat{\gamma}(s)=\gamma(0)=0$ for all $s \in[0, t]$. We have

$$
u(t, \omega) \geq g(0)-\mathcal{L}(\hat{\gamma}, \omega) \geq g(0)-t b .
$$

By (2.12), we also have the lower bound

$$
\mathcal{L}(\gamma, \omega) \geq t K\left(\frac{\gamma(t)-\gamma(0)}{t}\right)+a t
$$

Since $\gamma \in M_{1}(t, \omega)$, we may combine these two estimates with $u(t, \omega) \leq 1+g(\gamma(t))-\mathcal{L}(\gamma, \omega)$ to conclude

$$
K\left(\frac{\gamma(t)-\gamma(0)}{t}\right) \leq \frac{1}{t}+(b-a)+\frac{g(\gamma(t))-g(0)}{t} \leq 1+(b-a)+C_{1}\left(\frac{1+|\gamma(t)|}{t}\right) .
$$

Since $K(p)$ grows super-linearly in $|p|$, (2.15) follows.

Next, consider $\gamma$ at integer times $k \in[1, t-1] \cap \mathbb{Z}$. We will show that there is a constant $R_{1}$, independent of $t>1$, such that at least one time $k \in[1, t-1] \cap \mathbb{Z}$ must satisfy both

$$
|\gamma(k)-\gamma(k-1)| \leq R_{1} \text { and }|\gamma(k+1)-\gamma(k)| \leq R_{1}
$$

Arguing by way of contradiction, let us suppose (2.17) does not hold. Then $|\gamma(j+1)-\gamma(j)|>R_{1}$ must hold for at least $t / 3$ of the times $j \in[1, t-1] \cap \mathbb{Z}$. This implies that

$$
u(t, \omega) \leq 1+g(\gamma(t))-\mathcal{L}(\gamma, \omega) \leq 1+g(\gamma(t))-a t-\frac{t}{3} \min _{|q| \geq R_{1}} K(q) .
$$

On the other hand, by Lemma 2.1 and (2.15), we know that

$$
u(t, \omega) \geq g(\gamma(t))-b t-t K\left(\frac{\gamma(t)-\gamma(0)}{t}\right)-1 \geq g(\gamma(t))-b t-t \max _{|q| \leq R_{0}} K(q)-1
$$

holds for all $\gamma \in M_{1}(t, \omega)$. Combining these two bounds we obtain

$$
\frac{1}{3} \min _{|q| \geq R_{1}} K(q) \leq 1+(b-a)+\max _{|q| \leq R_{0}} K(q) .
$$

If $R_{1}>R_{0}$ is sufficiently large (depending only on $b-a, R_{0}$, and $K$ ) this forces a contradiction. So, (2.17) must hold. 
Now we conclude the proof. Let $R_{2}>R_{1}$, and suppose that for some $t_{1}, t_{2} \in[0, t]$ with $1 \leq$ $\left|t_{2}-t_{1}\right| \leq 2$ we have $\left|\gamma\left(t_{2}\right)-\gamma\left(t_{1}\right)\right| \geq R_{2}$. Let $k \in[1, t-1] \cap \mathbb{Z}$ be such that (2.17) holds. Without loss of generality, we may suppose $k+1 \leq t_{1}<t_{2}$. Consider the path $\hat{\gamma}$ defined by

$$
\hat{\gamma}(s)=\left\{\begin{array}{cl}
\gamma(s), & \text { for } s \in[0, k-1] \cup\left[t_{2}, t\right], \\
\gamma(k-1)+(s-k+1)(\gamma(k+1)-\gamma(k-1)), & \text { for } s \in[k-1, k], \\
\gamma(s+1), & \text { for } s \in\left[k, t_{1}-1\right],
\end{array}\right.
$$

and for $s \in\left[t_{1}-1, t_{2}\right]$

$$
\hat{\gamma}(s)=\gamma\left(t_{1}\right)+\left(\gamma\left(t_{2}\right)-\gamma\left(t_{1}\right)\right) \frac{s-t_{1}+1}{t_{2}-t_{1}+1} .
$$

Then we have

$$
\begin{aligned}
\mathcal{L}(\hat{\gamma})-\mathcal{L}(\gamma) \leq & 4(b-a)+\int_{k-1}^{k} K\left(\hat{\gamma}^{\prime}(s)\right) d s+\int_{t_{1}-1}^{t_{2}} K\left(\hat{\gamma}^{\prime}(s)\right) d s \\
& -\int_{k-1}^{k+1} K\left(\gamma^{\prime}(s)\right) d s-\int_{t_{1}}^{t_{2}} K\left(\gamma^{\prime}(s)\right) d s \\
\leq & 4(b-a)+K(\gamma(k+1)-\gamma(k-1))+\left(t_{2}-t_{1}+1\right) K\left(\frac{\gamma\left(t_{2}\right)-\gamma\left(t_{1}\right)}{t_{2}-t_{1}+1}\right) \\
& -2 K\left(\frac{\gamma(k+1)-\gamma(k-1)}{2}\right)-\left(t_{2}-t_{1}\right) K\left(\frac{\gamma\left(t_{2}\right)-\gamma\left(t_{1}\right)}{t_{2}-t_{1}}\right) \\
\leq & M+\left(t_{2}-t_{1}+1\right) K\left(\frac{\gamma\left(t_{2}\right)-\gamma\left(t_{1}\right)}{t_{2}-t_{1}+1}\right)-\left(t_{2}-t_{1}\right) K\left(\frac{\gamma\left(t_{2}\right)-\gamma\left(t_{1}\right)}{t_{2}-t_{1}}\right)
\end{aligned}
$$

where

$$
M=4(b-a)+\max _{|z| \leq 2 R_{1}} K(z) .
$$

Let $\Delta t=t_{2}-t_{1}$ and $\sigma=(\Delta t+1) /(\Delta t)$ and $z=\left(\gamma\left(t_{2}\right)-\gamma\left(t_{1}\right)\right) /\left(t_{2}-t_{1}+1\right)$. The inequality (2.18) has the form

$$
\mathcal{L}(\hat{\gamma})-\mathcal{L}(\gamma) \leq M+(\Delta t+1) K(z)-\Delta t K(\sigma z)
$$

The properties of $K$ (convexity and super-linear growth) imply that if $R_{2}$ sufficiently large, then

$$
\inf _{|z| \geq R_{2} / 3} K(\sigma z)-\sigma K(z)>M+1 .
$$

Applying this at (2.19) we conclude $\mathcal{L}\left(\gamma^{\prime}\right)-\mathcal{L}(\gamma)<1$, which contradicts the fact that $\gamma \in M_{1}(t, \omega)$. Therefore, we must have $\left|\gamma\left(t_{2}\right)-\gamma\left(t_{1}\right)\right| \leq R_{2}$ if $1 \leq\left|t_{1}-t_{2}\right| \leq 2$. This and the triangle inequality now imply the desired result for all $t_{1}, t_{2} \in[0, t]$.

\section{Important cubes}

Our method of estimating the variance of $u$ involves bounding the random variable $\left|\sigma_{j} u-u\right|$. So, we must understand when changing the value of $\omega_{j}$ leads to a large change in the value of $u(t, \omega)$. Given a path $\gamma \in \mathcal{A}_{t}$ and an index $j \in \mathbb{Z}^{d}$, define

$$
\pi_{j}(\gamma)=\left|\left\{s \in[0, t] \mid \gamma(s) \in Q_{j}\right\}\right|,
$$

which is the total time that the path $\gamma$ occupies the cube $Q_{j}$. Observe that for any path $\gamma \in \mathcal{A}_{t}$, we have

$$
\mathcal{L}\left(\gamma, \phi_{j} \omega\right) \leq \mathcal{L}(\gamma, \omega)+\left(b-\omega_{j}\right) \pi_{j}(\gamma)
$$


In particular, if $\gamma \in M_{\delta}(t, \omega)$, then

$$
\begin{aligned}
\sigma_{j} u(t, \omega)=u\left(t, \phi_{j} \omega\right) & \geq g(\gamma(t))-\mathcal{L}\left(\gamma, \phi_{j} \omega\right) \\
& \geq g(\gamma(t))-\mathcal{L}(\gamma, \omega)-\left(b-\omega_{j}\right) \pi_{j}(\gamma) \\
& \geq u(t, \omega)-\left(b-\omega_{j}\right) \pi_{j}(\gamma)-\delta
\end{aligned}
$$

From this we deduce that if $\omega_{j}=b$ or if there is $\gamma \in M_{\delta}(t, \omega)$ for which $\pi_{j}(\gamma)=0$, then it must be true that $u(t, \omega)-\sigma_{j} u(t, \omega) \leq \delta$. On the other hand, this also shows that if $u(t, \omega)-\sigma_{j} u(t, \omega)>\delta$, then $\omega_{j}=a$ and $\pi_{j}(\gamma)>0$ must hold for all $\gamma \in M_{\delta}(t, \omega)$. This motivates the following definition. We say that the cube $Q_{j}$ is important if $\omega_{j}=a$ and for some $\delta>0$ we have

$$
\pi_{j}(\gamma)>0, \quad \forall \gamma \in M_{\delta}(t, \omega)
$$

Observe that if (2.22) holds for some $\delta>0$, then it also holds for all $\delta^{\prime} \in(0, \delta]$. So, $Q_{j}$ is important if $\omega_{j}=a$ and for $\delta$ sufficiently small every $\delta$-approximate optimizer spends time in cube $Q_{j}$. Let $\mathcal{I}_{j} \subset \Omega$ denote the event that the cube $Q_{j}$ is important:

$$
\begin{aligned}
\mathcal{I}_{j} & =\left\{\omega \in \Omega \mid \omega_{j}=a ; \exists \delta>0 \text { such that } \pi_{j}(\gamma)>0 \forall \gamma \in M_{\delta}(t, \omega)\right\} \\
& =\bigcup_{n \geq 1}\left\{\omega \in \Omega \mid \omega_{j}=a ; \pi_{j}(\gamma)>0 \forall \gamma \in M_{1 / n}(t, \omega)\right\}
\end{aligned}
$$

The above analysis shows that

$$
\left\{\omega \in \Omega \mid u(t, \omega)>\sigma_{j} u(t, \omega)\right\} \subset \mathcal{I}_{j}, \quad \forall j \in \mathbb{Z}^{d}
$$

so we have

$$
\mathbb{P}\left(\sigma_{j} u<u\right) \leq \mathbb{P}\left(\mathcal{I}_{j}\right)
$$

Observe that $\mathbb{P}\left(\mathcal{I}_{j}\right)$ depends on $t$, in addition to $j$.

It will be useful to further classify some cubes as very important. To this end, we define a set of cubes

$$
N(\delta, \omega)=\bigcup_{\gamma \in M_{\delta}(\omega)}\left\{k \in \mathbb{Z}^{d} \mid \pi_{k}(\gamma)>0\right\}
$$

This is the set of all cubes visited by some path $\gamma \in M_{\delta}(t, \omega)$. Next, we define the event $\mathcal{I}_{j}^{+} \subset \mathcal{I}_{j} \subset \Omega$ that cube $Q_{j}$ is very important:

$$
\mathcal{I}_{j}^{+}=\left\{\omega \in \mathcal{I}_{j} \mid \exists \delta>0 \text { such that } \omega_{\ell}=b \quad \forall \ell \in N(\delta, \omega) \backslash\{j\}\right\} .
$$

On this event, $Q_{j}$ is an important cube, and for any other cube $Q_{\ell}$ visited by a path $\gamma \in M_{\delta}$, we have $\omega_{\ell}=b$, if $\delta$ is sufficiently small. On the event $\mathcal{I}_{j}^{-}=\mathcal{I}_{j} \backslash \mathcal{I}_{j}^{+}$, cube $Q_{j}$ is important but not very important: for any $\delta>0$ we can find a path $\gamma \in M_{\delta}(\omega)$ such that $\gamma$ passes through another cube $Q_{\ell} \neq Q_{j}$, on which $\omega_{\ell}=a$. The following lemma shows that the only way for $\left(u-\sigma_{j} u\right)^{2} 1_{\mathcal{I}_{j}}$ to be large is if $Q_{j}$ is very important.

Lemma 2.3 There is a constant $C_{0}>0$, depending only on $K$ and $|b-a|$, such that

$$
\mathbb{P}\left(\left\{\omega \mid\left(u-\sigma_{j} u\right)^{2} 1_{\mathcal{I}_{j}^{-}}<C_{0}\right\}\right)=1
$$

holds for all $t \geq 1$ and $j \in \mathbb{Z}^{d}$. 
Proof of Lemma 2.3; If $\omega_{j}=b$, then $\omega \notin \mathcal{I}_{j}^{-}$, so obviously $\left(u-\sigma_{j} u\right)^{2} 1_{\mathcal{I}_{j}^{-}}=0$. Hence, we may assume $\omega_{j}=a$ and $\omega \in \mathcal{I}_{j}^{-}$. When $\omega_{j}=a$, we clearly have $\sigma_{j} u \leq u$, since $\mathcal{L}\left(\gamma, \phi_{j} \omega\right) \geq \mathcal{L}(\gamma, \omega)$ in this case. So, we must bound $u-\sigma_{j} u$ from above.

Consider an approximate optimizer $\gamma \in M_{\delta}(\omega)$ for some $\delta \leq 1$. If $\pi_{j}(\gamma) \leq C$ then $u-\sigma_{j} u \leq$ $(b-a) C+\delta$ according to (2.21). So, we must consider the possibility that $\pi_{j}(\gamma)>0$ is large. Since $\omega \in \mathcal{I}_{j}^{-}$, we may assume the path $\gamma$ also passes through another cube $Q_{\ell}$, with $\ell \neq j$, for which $\omega_{\ell}=a$. We will construct a new path $\hat{\gamma}$ such that $\pi_{j}(\hat{\gamma}) \leq 1$ and $\mathcal{L}(\hat{\gamma}, \omega) \leq \mathcal{L}(\gamma, \omega)+C$. The two paths $\hat{\gamma}$ and $\gamma$ will have the same starting and ending points. This implies that the difference $u-\sigma_{j} u$ is bounded by a constant, since by (2.20) we have

$$
\begin{aligned}
\sigma_{j} u=u\left(t, \phi_{j} \omega\right) & \geq g(\hat{\gamma}(t))-\mathcal{L}\left(\hat{\gamma}, \phi_{j} \omega\right) \\
& \geq g(\hat{\gamma}(t))-\mathcal{L}(\hat{\gamma}, \omega)-(b-a) \pi_{j}(\hat{\gamma}) \\
& \geq g(\hat{\gamma}(t))-\mathcal{L}(\gamma, \omega)-C-(b-a) \pi_{j}(\hat{\gamma}) \geq u(t, \omega)-C-(b-a)-\delta .
\end{aligned}
$$

Suppose that $\left[t_{1}, t_{2}\right]$ is the smallest interval containing all $s$ for which $\gamma(s) \in Q_{j}$. We may assume $t_{2}-t_{1} \geq \pi_{j}(\gamma)>1$. Suppose that $\gamma\left(t_{3}\right) \in Q_{\ell}$ where $\ell \neq j$ and $\omega_{\ell}=a$. We may suppose that $t_{3}>t_{2}$ (the case $t_{3}<t_{1}$ is similar). Define the new path $\hat{\gamma}$ as follows:

(i) For $s \in\left[0, t_{1}\right]$, let $\hat{\gamma}(s)=\gamma(s)$.

(ii) For $s \in\left[t_{1}, t_{1}+1\right]$, let $\hat{\gamma}(s)=\gamma\left(t_{1}\right)+\left(s-t_{1}\right)\left(\gamma\left(t_{2}\right)-\gamma\left(t_{1}\right)\right)$.

(iii) For $s \in\left[t_{1}+1, t_{1}+1+\left(t_{3}-t_{2}\right)\right]$, let $\hat{\gamma}(s)=\gamma\left(s-t_{1}-1+t_{2}\right)$.

(iv) For $s \in\left[t_{1}+1+\left(t_{3}-t_{2}\right)\right.$, $\left.t_{3}\right]$, let $\hat{\gamma}(s)=\gamma\left(t_{3}\right)$.

(v) For $s \in\left[t_{3}, t\right]$, let $\hat{\gamma}(s)=\gamma(s)$.

Much of $\hat{\gamma}$ is just a linear reparameterization of $\gamma$, and we have

$$
\int_{0}^{t} L\left(\hat{\gamma}^{\prime}(s), \hat{\gamma}(s), \omega\right) d s-\int_{0}^{t} L\left(\gamma^{\prime}(s), \gamma(s), \omega\right) d s \leq \int_{t_{1}}^{t_{1}+1} K\left(\hat{\gamma}^{\prime}(s)\right) d s \leq K\left(\gamma\left(t_{2}\right)-\gamma\left(t_{1}\right)\right) .
$$

Since $\left|\gamma\left(t_{2}\right)-\gamma\left(t_{1}\right)\right|$ is bounded by the diameter of cube $Q_{j}$, we have $\mathcal{L}\left(\gamma^{\prime}, \omega\right) \leq \mathcal{L}(\gamma, \omega)+C$.

\section{Proof of Theorem 1.1}

In this section we prove Theorem 1.1. As we have mentioned, the main argument is similar to that of [4]. In particular, it is convenient to average $u(t, \omega)$ over a random shift of the environment.

\section{Random shifting of the environment}

We now consider an augmented probability space $\tilde{\Omega}=\Omega \times \Omega_{1}$ with product measure $\tilde{\mathbb{P}}=\mathbb{P} \times P_{1}$, and we introduce a random function $h\left(\omega_{1}\right): \Omega_{1} \rightarrow \mathbb{Z}^{d}$ to define a random shift of the environment. For $\left(\omega, \omega_{1}\right) \in \tilde{\Omega}$, let us define

$$
\tilde{u}\left(t, \omega, \omega_{1}\right)=u\left(t, \tau_{h\left(\omega_{1}\right)} \omega\right)=\sup _{\gamma \in \mathcal{A}_{t}} g(\gamma(t))-\mathcal{L}\left(\gamma+h\left(\omega_{1}\right), \omega\right)
$$

where $\gamma+h\left(\omega_{1}\right)$ denotes the shifted path $t \mapsto \gamma(t)+h\left(\omega_{1}\right)$. We define $M_{\delta}\left(\omega, \omega_{1}\right)=M_{\delta}\left(\tau_{h\left(\omega_{1}\right)} \omega\right)$ to be the set of paths $\gamma \in \mathcal{A}_{t}$ for which

$$
\tilde{u}\left(t, \omega, \omega_{1}\right) \leq g(\gamma(t))-\mathcal{L}\left(\gamma+h\left(\omega_{1}\right), \omega\right)+\delta .
$$


We construct $\Omega_{1}, P_{1}$, and $h$ in such a way that $\left|\tilde{u}\left(t, \omega, \omega_{1}\right)-u(t, \omega)\right|=o(\sqrt{t})$, and for this reason an estimate of $\operatorname{var}(\tilde{u})$ that is sublinear in $t$ will imply a sublinear bound for $\operatorname{var}(u)$.

The random shift $h\left(\omega_{1}\right)$ will lie in the set $[0, m)^{d} \subset \mathbb{R}^{d}$ where $m=\lfloor t \zeta$, for some positive $\zeta<1 / 2$. For $d \geq 3$, it will suffice to choose $\zeta \in\left(\frac{1}{d}, \frac{1}{2}\right)$. For $d=2$, we will require that $\zeta \in\left(\frac{\nu-1}{2 \nu-1}, 1 / 2\right)$, where $\nu$ was defined by the non-degeneracy condition (1.6). Denote by $P_{0}$ the product probability measure on the set $\Omega_{0}=\{a, b\}^{m^{2}}$ and having marginal distribution $P_{0}^{\prime}(a)=\alpha, P_{0}^{\prime}(b)=\beta=1-\alpha \in(0,1)$. The following statement is Lemma 3 from [4], so we omit the proof:

Lemma 3.1 There exists a constant $C>0$ independent of $m=\left\lfloor t^{\zeta}\right\rfloor$, and a function $\tilde{h}: \Omega_{0} \rightarrow$ $\{0,1, \ldots, m-1\}$ for which the following two conditions hold:

(i) $P_{0}(\tilde{h}=i) \leq \frac{C}{m}$ for all $i \in[0, m-1]$, and

(ii) for every $x, y \in \Omega_{0}$ that differ in at most one coordinate, the difference between $\tilde{h}(x)$ and $\tilde{h}(y)$ satisfies

$$
|\tilde{h}(x)-\tilde{h}(y)| \leq 1
$$

Define the set $\Theta=\Theta_{t}=\{1,2, \ldots, d\} \times\left\{1,2, \ldots, m^{2}\right\}$. Let $\Omega_{1}=\{a, b\}^{\Theta}$, and let $P_{1}$ be a uniform probability measure on $\Omega_{1}$. Each $\omega_{1} \in \Omega_{1}$ can be written as $\omega_{1}=\left(\omega_{1}^{1}, \omega_{1}^{2}, \ldots, \omega_{1}^{d}\right)$, where each $\omega_{1}^{i}$ is a binary sequence of length $m^{2}$. Let $\vec{e}_{i}$ denote the $i$-th coordinate vector. Define $h\left(\omega_{1}\right)=\sum_{i=1}^{d} \tilde{h}\left(\omega_{1}^{i}\right) \vec{e}_{i}$. There exists a constant $C>0$ independent on $m$ such that for each $x \in\{0,1, \ldots, m-1\}^{d}$ one has $P_{1}(h=x) \leq \frac{C}{m^{d}}$. Moreover, if $\omega_{1}$ and $\omega_{1}^{\prime}$ differ in exactly one coordinate then we have $\mid h\left(\omega_{1}\right)-$ $h\left(\omega_{1}^{\prime}\right) \mid \leq 1$. Given the space $\tilde{\Omega}=\Omega \times \Omega_{1}$ with the product measure $\tilde{\mathbb{P}}=\mathbb{P} \times P_{1}$ on $\Omega \times \Omega_{1}$ defined in this way, we now consider the function $\tilde{u}$ defined by (3.29).

Lemma 3.2 There is a constant $C>0$ such that

$$
\left|u(t, \omega)-\tilde{u}\left(t, \omega, \omega_{1}\right)\right| \leq C\left|h\left(\omega_{1}\right)\right| \leq C t^{\zeta}, \quad \forall t>1
$$

holds $\tilde{\mathbb{P}}$-almost surely, and

$$
\operatorname{var} u \leq C \operatorname{var} \tilde{u}+C t^{2 \zeta}, \quad \forall t>1 .
$$

Proof of Lemma 3.2; We will prove that $\left|u(t, \omega)-\tilde{u}\left(t, \omega, \omega_{1}\right)\right| \leq C\left|h\left(\omega_{1}\right)\right|$, $\tilde{\mathbb{P}}_{\text {-almost surely, for }}$ some constant $C>0$ independent of $m$ and $t$. Given a path $\gamma \in M_{\delta}(\omega)$, we can modify it to construct an approximate optimizer for $\tilde{u}\left(t, \omega, \omega_{1}\right)$, thus estimating $\tilde{u}\left(t, \omega, \omega_{1}\right)-u(t, \omega)$ from above. However, we cannot simply shift $\gamma$ by $-h\left(\omega_{1}\right)$, since we must preserve the starting and ending points.

Suppose $\left|h\left(\omega_{1}\right)\right| \leq \sqrt{d} \kappa$, with $\kappa \in[1, m] \cap \mathbb{Z}$. Fixing a path $\gamma \in M_{\delta}(\omega)$, we define the new path $\hat{\gamma}$ in the following way:

(i) For $r \in[0, \kappa]$, set $\hat{\gamma}(r)=\gamma(0)+\left(\frac{r}{\kappa}\right)\left(\gamma(2 \kappa)-h\left(\omega_{1}\right)-\gamma(0)\right)$.

(ii) For $r \in[\kappa, t-\kappa]$, set $\hat{\gamma}(r)=\gamma(r+\kappa)-h\left(\omega_{1}\right)$.

(iii) For $r \in[t-\kappa, t]$, set $\hat{\gamma}(r)=\gamma(t)+(r-t) \frac{h\left(\omega_{1}\right)}{\kappa}$. 
We now verify that the path $\hat{\gamma}$ yields the desired bound on $\tilde{u}\left(t, \omega, \omega_{1}\right)-u(t, \omega)$ and var $u$. Since $\gamma \in M_{\delta}(\omega)$, we have

$$
\begin{aligned}
\tilde{u}\left(t, \omega, \omega_{1}\right)-u(t, \omega) \geq & -\int_{0}^{\kappa} L\left(\hat{\gamma}^{\prime}(s), \hat{\gamma}(s)+h\left(\omega_{1}\right), \omega\right) d s-\int_{t-\kappa}^{t} L\left(\hat{\gamma}^{\prime}(s), \hat{\gamma}(s)+h\left(\omega_{1}\right), \omega\right) d s \\
& +\int_{0}^{2 \kappa} L\left(\gamma^{\prime}(s), \gamma(s), \omega\right) d s-\delta \\
\geq & -C \kappa\left(1+\sup _{|z| \leq 2 R} K(z)\right)-\delta,
\end{aligned}
$$

where $C$ is a positive real number that depends only on $K,|b-a|$. In a similar way we prove that $\tilde{u}\left(t, \omega, \omega_{1}\right)-u(t, \omega) \leq C \kappa+\delta$. Recalling that $m=\left\lfloor t^{\zeta}\right\rfloor$, we obtain (3.30). Therefore

$$
\begin{aligned}
\operatorname{var} u & =\tilde{\mathbb{E}}\left[(u-\tilde{\mathbb{E}}(u))^{2}\right] \\
& =\tilde{\mathbb{E}}\left[(\tilde{u}-\tilde{\mathbb{E}}(\tilde{u})+(u-\tilde{u})-\tilde{\mathbb{E}}(u-\tilde{u}))^{2}\right] \\
& \leq 3 \operatorname{var} \tilde{u}+3 \tilde{\mathbb{E}}\left[((u-\tilde{u})-\tilde{\mathbb{E}}(u-\tilde{u}))^{2}\right] \\
& \leq 3 \operatorname{var} \tilde{u}+12 C^{2} m^{2},
\end{aligned}
$$

which is (3.31).

\section{Variance estimate for $\tilde{u}$}

Given Lemma 3.2 and the choice of $\zeta<1 / 2$, we now wish to establish a bound of order $t / \log t$ for the variance of $\tilde{u}\left(t, \omega, \omega_{1}\right)$ under $\tilde{\mathbb{P}}$. The augmented probability space was constructed in such a way that $\tilde{u}\left(t, \omega, \omega_{1}\right)$ is amenable to Talagrand's inequality. The function $u$ depends on $\omega_{j}$ for only $O\left(t^{d}\right)$ of the indices $j \in \mathbb{Z}^{d}$ :

Lemma 3.3 There is a constant $R>0$ such that

$$
\tilde{u}\left(t, \omega, \omega_{1}\right)=\tilde{u}\left(t, \phi_{j} \omega, \omega_{1}\right), \quad \forall j \in \mathbb{Z}^{d},|j|>R t, \quad t>0
$$

holds $\tilde{P}$ almost surely.

Proof: Since $\left|h\left(\omega_{1}\right)\right| \leq m \sqrt{d}$, this is a consequence of Lemma 2.2, no approximate optimizer passes through cube $j$, if $R$ is sufficiently large and $|j|>R t$.

In view of Lemma 3.3, we may regard $\tilde{u}$ as a function of no more than $C t^{d}+d m^{2}$ random variables taking values in the set $\{a, b\}$. In this way, Talagrand's inequality (Theorem 1.2) implies that there is a constant $C>0$, independent of $t>0$, such that

$$
\operatorname{var}(\tilde{u}) \leq C \sum_{j \in B_{t}} \frac{\left\|\rho_{j} \tilde{u}\right\|_{2}^{2}}{1+\log \frac{\left\|\rho_{j} \tilde{u}\right\|_{2}}{\left\|\rho_{j} \tilde{u}\right\|_{1}}}+C \sum_{k \in \Theta_{t}} \frac{\left\|\rho_{k} \tilde{u}\right\|_{2}^{2}}{1+\log \frac{\left\|\rho_{k} \tilde{u}\right\|_{2}}{\left\|\rho_{k} \tilde{u}\right\|_{1}}}
$$


where $B_{t}$ is the set $B_{t}=\left\{j \in \mathbb{Z}^{d}|| j \mid \leq R t\right\}$, whose cardinality is bounded by $C t^{d}$. The norms $\|\cdot\|_{2}$ and $\|\cdot\|_{1}$ refer to the $L^{2}(\tilde{\Omega}, \tilde{P})$ and $L^{1}(\tilde{\Omega}, \tilde{P})$ norms, respectively. Observe that if $k \in \Theta_{t}$, then $\rho_{k} \tilde{f}$ corresponds to translation of the random environment:

$$
\rho_{k} \tilde{u}=\frac{\sigma_{k} \tilde{u}-\tilde{u}}{2}=\frac{\tilde{u}\left(t, \omega, \phi_{k} \omega_{1}\right)-\tilde{u}\left(t, \omega, \omega_{1}\right)}{2} .
$$

If $j \in B_{t}$, then $\rho_{j} \tilde{u}$ corresponds to a local change in the random environment over the cube $Q_{j}$ :

$$
\rho_{j} \tilde{u}=\frac{\sigma_{j} \tilde{u}-\tilde{u}}{2}=\frac{\tilde{u}\left(t, \phi_{j} \omega, \omega_{1}\right)-\tilde{u}\left(t, \omega, \omega_{1}\right)}{2} .
$$

Let us first consider the second sum in (3.32). We will show that this sum is $O\left(t^{2 \zeta}\right)$.

Lemma 3.4 There is a constant $C>0$ such that

$$
\sum_{k \in \Theta_{t}} \frac{\left\|\rho_{k} \tilde{u}\right\|_{2}^{2}}{1+\log \frac{\left\|\rho_{k} \tilde{u}\right\|_{2}}{\left\|\rho_{k} \tilde{u}\right\|_{1}}} \leq C t^{2 \zeta}
$$

holds for all $t>1$.

Proof: Since there are only $\left|\Theta_{t}\right|=m^{2} \leq t^{2 \zeta}$ terms in the sum and since

$$
1+\log \frac{\left\|\rho_{k} \tilde{u}\right\|_{2}}{\left\|\rho_{k} \tilde{u}\right\|_{1}} \geq 1
$$

the lemma will follow from a uniform bound on $\left\|\rho_{k} \tilde{u}\right\|_{2}$. By definition of $h\left(\omega_{1}\right)$, we know that $\left|h\left(\phi_{k} \omega_{1}\right)-h\left(\omega_{1}\right)\right| \leq 1$. So, by Lemma 3.2. we have $\left|\tilde{u}\left(t, \omega, \omega_{1}\right)-\tilde{u}\left(t, \omega, \phi_{k} \omega_{1}\right)\right| \leq C\left|h\left(\phi_{k} \omega_{1}\right)-h\left(\omega_{1}\right)\right| \leq$ $C$ holds $\tilde{P}$ almost surely, for all $k \in \Theta_{t}, t \geq 1$.

Having established (3.33), we now consider the first sum in (3.32).

Proposition 3.5 There is a constant $C>0$ such that

$$
\sum_{j \in B_{t}} \frac{\left\|\rho_{j} \tilde{u}\right\|_{2}^{2}}{1+\log \frac{\left\|\rho_{j} \tilde{u}\right\|_{2}}{\left\|\rho_{j} \tilde{u}\right\|_{1}}} \leq C \frac{t}{\log t}
$$

holds for all $t>1$.

Since we may have $\alpha \neq \beta$, we will make use of the following fact, proved in the appendix:

Lemma 3.6 Let $C^{\prime}=\min \left\{\frac{\alpha}{\beta}, \frac{\beta}{\alpha}\right\}$ and $C^{\prime \prime}=\max \left\{\frac{\alpha}{\beta}, \frac{\beta}{\alpha}\right\}$. For any measureable set $A \subset \Omega$,

$$
C^{\prime} \mathbb{P}(A) \leq \mathbb{P}\left(\phi_{j} A\right) \leq C^{\prime \prime} \mathbb{P}(A)
$$

holds for all $j \in \mathbb{Z}^{d}$. Also, for every nonnegative integrable $\psi$, we have

$$
C^{\prime} \mathbb{E}\left(\psi \circ \phi_{j}\right) \leq \mathbb{E}(\psi) \leq C^{\prime \prime} \mathbb{E}\left(\psi \circ \phi_{j}\right) .
$$


Proof of Proposition 3.5. Let us begin by estimating $\left\|\rho_{j} \tilde{u}\right\|_{2}^{2}$. By Lemma 3.6, we have

$$
\left\|\rho_{j} \tilde{u}\right\|_{2}^{2}=\tilde{\mathbb{E}}\left[\left(\sigma_{j} \tilde{u}-\tilde{u}\right)^{2} 1_{\sigma_{j} \tilde{u}>\tilde{u}}\right]+\tilde{\mathbb{E}}\left[\left(\sigma_{j} \tilde{u}-\tilde{u}\right)^{2} 1_{\sigma_{j} \tilde{u}<\tilde{u}}\right] \leq C \tilde{\mathbb{E}}\left[\left(\sigma_{j} \tilde{u}-\tilde{u}\right)^{2} 1_{\sigma_{j} \tilde{u}<\tilde{u}}\right] .
$$

Recalling the definition (2.23), let $\tilde{\mathcal{I}}_{j} \subset \tilde{\Omega}$ be the event that $Q_{j}$ is an important cube in the shifted environment:

$$
\tilde{\mathcal{I}}_{j}=\left\{\left(\omega, \omega_{1}\right) \in \tilde{\Omega} \mid \tau_{h\left(\omega_{1}\right)} \omega \in \mathcal{I}_{j}\right\} .
$$

Because of (2.25), the event $\left\{\sigma_{j} \tilde{u}<\tilde{u}\right\}$ is contained in the event $\tilde{\mathcal{I}}_{j}$. So, we have

$$
\left\|\rho_{j} \tilde{u}\right\|_{2}^{2} \leq C \tilde{\mathbb{E}}\left[\left(\sigma_{j} \tilde{u}-\tilde{u}\right)^{2} 1_{\tilde{\mathcal{I}}_{j}}\right] .
$$

The difference $\left|\sigma_{j} \tilde{u}-\tilde{u}\right|$ could be large in some cases, even on the event $\tilde{\mathcal{I}}_{j}$, so we will distinguish a few possible scenarios. Let $\tilde{\mathcal{I}}_{j}^{+} \subset \tilde{\mathcal{I}}_{j}$ denote the event that cube $Q_{j}$ is very important in the shifted environment:

$$
\tilde{\mathcal{I}}_{j}^{+}=\left\{\left(\omega, \omega_{1}\right) \in \tilde{\mathcal{I}}_{j} \mid \tau_{h\left(\omega_{1}\right)} \omega \in \mathcal{I}_{j}^{+}\right\} .
$$

Similarly, let $\tilde{\mathcal{I}}_{j}^{-}=\tilde{\mathcal{I}}_{j} \backslash \tilde{\mathcal{I}}_{j}^{+}$be the event that the cube $Q_{j}$ is important but not very important. Since $\omega \mapsto \tau_{h\left(\omega_{1}\right)} \omega$ is measure preserving on $\Omega$, we have

$$
\tilde{\mathbb{P}}\left(\left\{\left(\omega, \omega_{1}\right) \in \tilde{\Omega} \mid\left(\tilde{u}-\sigma_{j} \tilde{u}\right)^{2} 1_{\tilde{\mathcal{I}}_{j}^{-}}>C_{0}\right\}\right)=\mathbb{P}\left(\left\{\omega \in \Omega \mid\left(u-\sigma_{j} u\right)^{2} 1_{\mathcal{I}_{j}^{-}}>C_{0}\right\}\right) .
$$

Consequently, from Lemma 2.3 and (3.37) we have

$$
\begin{aligned}
\left\|\rho_{j} \tilde{u}\right\|_{2}^{2} & \leq C \tilde{\mathbb{E}}\left[\left(\sigma_{j} \tilde{u}-\tilde{u}\right)^{2} 1_{\tilde{\mathcal{I}}_{j}^{+}}\right]+C \tilde{\mathbb{E}}\left[\left(\sigma_{j} \tilde{u}-\tilde{u}\right)^{2} 1_{\tilde{\mathcal{I}}_{j}^{-}}\right] \\
& \leq C \tilde{\mathbb{E}}\left[\left(\sigma_{j} \tilde{u}-\tilde{u}\right)^{2} 1_{\tilde{\mathcal{I}}_{j}^{+}}\right]+C C_{0} \tilde{\mathbb{P}}\left(\tilde{\mathcal{I}}_{j}\right) .
\end{aligned}
$$

Whether the event $\tilde{\mathcal{I}}_{j}^{+}$has small probability depends on the function $g(y)$, so we distinguish two cases. Let $\tilde{G} \subset \tilde{\Omega}$ denote the even that

$$
|\gamma(t)-\gamma(0)| \geq t^{1 / 4}, \quad \forall \gamma \in M_{1}\left(\omega, \omega_{1}\right) .
$$

On this event, all approximate minimizers must travel a distance at least $O\left(t^{1 / 4}\right)$ from their starting point $\gamma(0)=0$. According to the following lemma, the probability that minimizers travel that far when a cube $Q_{j}$ is very important must be small.

Lemma 3.7 There are constants $\kappa_{1}, \kappa_{2}>0$ such that

$$
\tilde{\mathbb{P}}\left(\tilde{\mathcal{I}}_{j}^{+} \cap \tilde{G}\right) \leq \kappa_{1} e^{-\kappa_{2} t^{1 / 4}}, \quad \forall t>0, \quad j \in \mathbb{Z}^{d} .
$$

Therefore, returning to (3.38) and using the fact that $\left|\sigma_{j} \tilde{u}-\tilde{u}\right| \leq O(t)$ and $\tilde{\mathcal{I}}_{j}^{+}=\left(\tilde{\mathcal{I}}_{j}^{+} \cap \tilde{G}\right) \cup$ $\left(\tilde{\mathcal{I}}_{j}^{+} \cap \tilde{G}^{C}\right)$, we conclude

$$
\left\|\rho_{j} \tilde{u}\right\|_{2}^{2} \leq C t^{2} e^{-\kappa_{2} t^{1 / 4}}+C \tilde{\mathbb{P}}\left(\tilde{\mathcal{I}}_{j}\right)+C \tilde{\mathbb{E}}\left[\left(\sigma_{j} \tilde{u}-\tilde{u}\right)^{2} 1_{\tilde{\mathcal{I}}_{j}^{+} \cap \tilde{G}^{C}}\right] .
$$

Hence,

$$
\sum_{j \in B_{t}}\left\|\rho_{j} \tilde{u}\right\|_{2}^{2} \leq C\left|B_{t}\right| t^{2} e^{-\kappa_{2} t}+C \tilde{\mathbb{E}}\left[\sum_{j \in B_{t}} 1_{\tilde{\mathcal{I}}_{j}}\right]+C \tilde{\mathbb{E}}\left[\sum_{j \in B_{t}}\left(\sigma_{j} \tilde{u}-\tilde{u}\right)^{2} 1_{\tilde{\mathcal{I}}_{j}^{+} \cap \tilde{G}^{C}}\right] .
$$

With probability one, the sum $\sum_{j \in B_{t}} 1_{\tilde{\mathcal{I}}_{j}}$ is bounded by $O(t)$ because there can be at most $O(t)$ important cubes, as the total number of cubes visited is $O(t)$, by Lemma 2.2 ,

The last term in (3.39) is bounded as follows. First, 
Lemma 3.8 There are $\kappa_{1}, \kappa_{2}>0$ such that

$$
\tilde{\mathbb{P}}\left(\left\{\left(\omega, \omega_{1}\right) \mid\left(\sigma_{j} \tilde{u}-\tilde{u}\right)^{2} 1_{\tilde{G}^{C}}>C_{0} t^{1 / 2}\right\}\right) \leq \kappa_{1} e^{-\kappa_{2} t^{1 / 4}}
$$

holds for all $t>1$ and all $j \in \mathbb{Z}^{d}$.

Furthermore, if $\omega \in \mathcal{I}_{j}^{+}$, then $\omega \notin \mathcal{I}_{k}^{+}$for any $k \neq j$, since $Q_{j}$ must be the only important cube. Therefore, since $\left|\sigma_{j} \tilde{u}-\tilde{u}\right| \leq|b-a| t$ always holds (by (2.21) $)$, we must have

$$
\tilde{\mathbb{E}}\left[\sum_{j \in B_{t}}\left(\sigma_{j} \tilde{u}-\tilde{u}\right)^{2} 1_{\tilde{\mathcal{I}}_{j}^{+} \cap \tilde{G}^{C}}\right] \leq C_{0} t^{1 / 2}+|b-a|^{2} t^{2} \kappa_{1} e^{-\kappa_{2} t^{1 / 4}} .
$$

Considering (3.39), we have now shown that there is a constant $C^{\prime}>0$ for which

$$
\sum_{j \in B_{t}}\left\|\rho_{j} \tilde{u}\right\|_{2}^{2} \leq C^{\prime} t
$$

holds for all $t>1$.

Next we consider the denominator in (3.34). We show that there is a constant $C^{\prime \prime}>0$ such that

$$
\log \frac{\left\|\rho_{j} \tilde{u}\right\|_{2}}{\left\|\rho_{j} \tilde{u}\right\|_{1}} \geq C^{\prime \prime} \log t
$$

for all $t>1$. By the Cauchy-Schwarz inequality we see that

$$
\left\|\rho_{j} \tilde{u}\right\|_{1}=\left\|\rho_{j} \tilde{u} \cdot 1_{\sigma_{j} \tilde{u} \neq \tilde{u}}\right\|_{1} \leq\left\|\rho_{j} \tilde{u}\right\|_{2} \cdot \sqrt{\mathbb{P}\left(\sigma_{j} \tilde{u} \neq \tilde{u}\right)} .
$$

Since $\sigma_{j} \sigma_{j} u=u$, Lemma 3.6 implies

$$
\tilde{\mathbb{P}}\left(\sigma_{j} \tilde{u} \neq \tilde{u}\right)=\tilde{\mathbb{P}}\left(\sigma_{j} \tilde{u}>\tilde{u}\right)+\tilde{\mathbb{P}}\left(\sigma_{j} \tilde{u}<\tilde{u}\right) \leq\left(1+C^{\prime \prime}\right) \tilde{\mathbb{P}}\left(\sigma_{j} \tilde{u}<\tilde{u}\right) .
$$

Hence,

$$
\frac{\left\|\rho_{j} \tilde{u}\right\|_{2}}{\left\|\rho_{j} \tilde{u}\right\|_{1}} \geq \frac{1}{\sqrt{\left(1+C^{\prime \prime}\right) \tilde{\mathbb{P}}\left(\sigma_{j} \tilde{u}<\tilde{u}\right)}} .
$$

Therefore, to bound $\log \left(\left\|\rho_{j} \tilde{u}\right\|_{2} /\left\|\rho_{j} \tilde{u}\right\|_{1}\right)$ from below, we should find an upper bound for $\tilde{\mathbb{P}}\left(\sigma_{j} \tilde{u}<\tilde{u}\right)$. Because of (2.26), we know that

$$
\tilde{\mathbb{P}}\left(\sigma_{j} \tilde{u}<\tilde{u}\right) \leq \tilde{\mathbb{P}}\left(\tilde{\mathcal{I}}_{j}\right) .
$$

To estimate $\tilde{\mathbb{P}}\left(\tilde{\mathcal{I}}_{j}\right)$ we average in $\omega_{1}$, as was done in [4]:

$$
\begin{aligned}
\tilde{\mathbb{P}}\left(\tilde{\mathcal{I}}_{j}\right)=\tilde{\mathbb{E}}\left[1_{\tilde{\mathcal{I}}_{j}}\right] & =\tilde{\mathbb{E}}\left[\tilde{\mathbb{E}}\left[1_{\tilde{\mathcal{I}}_{j}} \mid \omega\right]\right] \\
& =\mathbb{E}\left[\sum_{z \in[0, m-1]^{d}} \tilde{\mathbb{E}}\left[1_{\tilde{\mathcal{I}}_{j}} \mid \omega, h\left(\omega_{1}\right)=z\right] P_{1}\left(h\left(\omega_{1}\right)=z\right)\right] .
\end{aligned}
$$

Observe that $\left(\omega, \omega_{1}\right) \in \tilde{\mathcal{I}}_{j}$ if and only if there is $\delta>0$ such that

$$
\pi_{j}(\gamma)>0 \text { for all } \gamma \in \tilde{M}_{\delta}\left(\omega, \omega_{1}\right)
$$


which holds if and only if for some $\delta>0$

$$
\pi_{j-z}(\gamma)>0 \quad \text { for all } \gamma \in M_{\delta}\left(t, \tau_{z} \omega\right), \quad z=h\left(\omega_{1}\right)
$$

So, for $\mathcal{I}_{j}$ defined by (2.24), we have

$$
\tilde{\mathbb{E}}\left[1_{\tilde{\mathcal{I}}_{j}} \mid \omega, h\left(\omega_{1}\right)=z\right]=1_{\mathcal{I}_{j-z}}\left(\tau_{z} \omega\right) .
$$

By Lemma 3.1, we also know that $P_{1}\left(h\left(\omega_{1}\right)=z\right) \leq C m^{-d}$ for any $z \in[0, m-1]^{d}$. Therefore,

$$
\begin{aligned}
\tilde{\mathbb{P}}\left(\tilde{\mathcal{I}}_{j}\right) & \leq C m^{-d} \mathbb{E}\left[\sum_{z} \tilde{\mathbb{E}}\left[1_{\mathcal{I}_{j}} \mid \omega, h\left(\omega_{1}\right)=z\right]\right]=C m^{-d} \mathbb{E}\left[\sum_{z} 1_{\mathcal{I}_{j-z}}\left(\tau_{z} \omega\right)\right] \\
& =C m^{-d} \mathbb{E}\left[\sum_{z} 1_{\mathcal{I}_{j-z}}(\omega)\right] .
\end{aligned}
$$

The last equality follows from the stationarity of $\mathbb{P}$ with respect to $\tau_{z}$. Now, given $\omega \in \Omega$, the sum

$$
\sum_{z \in[0, m-1]^{d}} 1_{\mathcal{I}_{j-z}}(\omega)
$$

counts the number of important cubes within the box $j-[0, m-1]^{d}$. These cubes are visited by all paths $\gamma \in M_{\delta}(\omega)$ for some $\delta>0$ sufficiently small. Hence, $\tilde{\mathbb{P}}\left(\tilde{\mathcal{I}}_{j}\right) \leq C m^{-d} \mathbb{E}\left[\# \Lambda_{j}\right]$ where

$$
\Lambda_{j}=\bigcup_{n \geq 1}\left\{k \in \mathbb{Z}^{d} \mid j-k \in[0, m-1]^{d}, \pi_{k}(\gamma)>0 \quad \forall \gamma \in M_{1 / n}(\omega)\right\} .
$$

We may interpret the random variable $\# \Lambda_{j}$ as the number of important cubes in the box $j-[0, m-1]^{d}$.

Obviously we have the trivial bound $\# \Lambda_{j} \leq O(t)$. This is because each path $\gamma \in M_{\delta}(\omega)$ has length $O(t)$, by Lemma 2.2, so $\pi_{k}(\gamma)>0$ for at most $O(t)$ indices $k$. Therefore,

$$
\mathbb{E}\left[\# \Lambda_{j}\right] \leq t \leq(m+1)^{1 / \zeta} .
$$

If $d \geq 3$, we may choose $\zeta \in(1 / d, 1 / 2)$, so that

$$
\tilde{\mathbb{P}}\left(\sigma_{j} \tilde{u}<\tilde{u}\right) \leq \tilde{\mathbb{P}}\left(\tilde{\mathcal{I}}_{j}\right) \leq C m^{-d} \mathbb{E}\left[\# \Lambda_{j}\right] \leq C m^{-d+1 / \zeta} \leq C t^{1-d \zeta}
$$

with $1-d \zeta<0$. If $d=2$, we need the following:

Lemma 3.9 Let $d=2$ and assume the non-degeneracy condition (1.6) holds. Then for each $p \in$ $\left(\frac{\nu-1+\zeta}{\zeta \nu}, 2\right)$ there exists a constant $C>0$ such that $\# \Lambda_{j} \leq C m^{p}$ holds with probability one, for all $j \in \mathbb{Z}^{d}$.

So, for $d=2$ we still have $\tilde{\mathbb{P}}\left(\sigma_{j} \tilde{u}<\tilde{u}\right) \leq C m^{-d} \mathbb{E}\left[\# \Lambda_{j}\right] \leq C n^{(p-2) / \zeta}$, with $(p-2) / \zeta<0$. Therefore, returning to (3.42) we conclude that there is a constant $C>0$ such that

$$
\log \frac{\left\|\rho_{j} \tilde{u}\right\|_{2}}{\left\|\rho_{j} \tilde{u}\right\|_{1}} \geq C \log t
$$

holds for all $t$ sufficiently large. Therefore, the proof of Proposition 3.5 is reduced to a proof of Lemma 3.7. Lemma 3.8 and, in case $d=2$, Lemma 3.9. These are proved in the next section.

Theorem 1.1 now follows immediately from (3.32), Lemma 3.4, and Proposition 3.5. 


\section{Proofs of the technical estimates}

\section{Proof of Lemma 3.7.}

Observe that

$$
\tilde{\mathbb{P}}\left(\tilde{\mathcal{I}}_{j}^{+} \cap \tilde{G}\right)=\mathbb{P}\left(\mathcal{I}_{j}^{+} \cap G\right)
$$

where $G \subset \Omega$ is the event for which

$$
|\gamma(t)-\gamma(0)| \geq t^{1 / 4}, \quad \forall \gamma \in M_{\delta}(t, \omega) .
$$

We will show that on the event $\mathcal{I}_{j}^{+} \cap G$, any approximate optimizer $\gamma \in M_{\delta}(\omega)$ must touch a set of $O\left(t^{1 / 4}\right)$ cubes which are almost uniformly spaced on a straight line segment of length $O(t)$ and on each of those cubes we have $V(x, \omega)=b$. Such an event can occur only with small probability.

Suppose $\omega \in \mathcal{I}_{j}^{+} \cap G$, and let $\gamma \in M_{\delta}(\omega)$ be such that (4.46) holds. Let $\left[t_{1}, t_{2}\right] \subset[0, t]$ be the smallest interval containing all $s$ for which $\gamma(s) \in Q_{j}$. Hence, $\omega(\gamma(s))=b$ for all $s \notin\left[t_{1}, t_{2}\right]$, since $\omega \in \mathcal{I}_{j}^{+}$. Since $\omega \in G$, we know that $|\gamma(t)-\gamma(0)| \geq t^{1 / 4}$, which means that either

$$
\left|\gamma\left(t_{1}\right)-\gamma(0)\right|>\frac{t^{1 / 4}}{3} \quad \text { or } \quad\left|\gamma(t)-\gamma\left(t_{2}\right)\right|>\frac{t^{1 / 4}}{3}
$$

must hold, because $\gamma\left(t_{1}\right), \gamma\left(t_{2}\right) \in \overline{Q_{j}}$. Let us assume that $\left|\gamma(t)-\gamma\left(t_{2}\right)\right|>\left(t^{1 / 4}\right) / 3$ holds; the other case is treated in a similar manner.

First, since $\omega(\gamma(s))=b$ for all $s \in\left(t_{2}, t\right]$, we may assume that $\gamma$ is a straight line between $\gamma\left(t_{2}\right)$ and $\gamma(t)$. Specifically, by redefining $\gamma$ slightly, we may assume that

$$
\gamma(s)=\gamma\left(t_{2}\right)+\frac{\gamma(t)-\gamma\left(t_{2}\right)}{t-t_{2}}\left(s-t_{2}\right), \quad \forall s \in\left[t_{2}, t\right],
$$

for otherwise, $\gamma$ would not be an optimal path. This follows from (2.12).

Next, given points $\gamma\left(t_{2}\right)$ and $\gamma(t)$, there is a unique pair $x_{t_{2}}, x_{t} \in \mathbb{Z}^{d}$ such that

$$
\gamma\left(t_{2}\right)=x_{t_{2}}+y_{t_{2}}, \quad \gamma(t)=x_{t}+y_{t}
$$

for some $y_{t_{2}}, y_{t} \in[0,1)^{d}$. Therefore, if we define the linear path

$$
\hat{\gamma}(s)=x_{t_{2}}+\frac{x_{t}-x_{t_{2}}}{t-t_{2}}\left(s-t_{2}\right), \quad s \in\left[t_{2}, t\right]
$$

we have $|\gamma(s)-\hat{\gamma}(s)| \leq 2 \sqrt{d}$ for $s \in\left[t_{2}, t\right]$. Therefore, for each $s \in\left[t_{2}, t\right]$ there must be a cube $Q_{\ell}$ such that dist $\left(\hat{\gamma}(s), Q_{\ell}\right) \leq 2 \sqrt{d}$ and $\omega\left(Q_{\ell}\right)=b$. For $y \in \mathbb{R}^{d}$, let $B_{y}$ denote the event that there is at least one cube $Q$ such that dist $(Q, y) \leq 2 \sqrt{d}$ and $\omega(Q)=b$. Then $\mathbb{P}\left(B_{y}\right)=1-\mathbb{P}\left(B_{y}^{C}\right) \leq 1-\alpha^{C_{3}}<1$, for a constant $C_{3}>0$ that depends only on the dimension $d$. Moreover, if $|y-z|>5 \sqrt{d}$, then $B_{y}$ and $B_{z}$ are independent events. Therefore, for fixed times $t_{2}<t$ and a fixed pair of points $x_{t_{2}}, x_{t} \in \mathbb{Z}^{d}$ satisfying $\left|x_{t_{2}}-x_{t}\right| \geq t^{1 / 4} / 2$ we have

$$
\mathbb{P}\left(\bigcap_{s \in\left[t_{2}, t\right]} B_{\hat{\gamma}(s)}\right) \leq\left(1-\alpha^{C_{3}}\right)^{C_{4} t^{1 / 4}}
$$

for some constant $C_{4}>0$ independent of $\epsilon$. 
By Lemma 2.2, we know there is a constant $R>0$ such that $|\gamma(s)-\gamma(0)| \leq t R$ for all $s \in[0, t]$. There are at most $O\left(t^{2 d}\right)$ possible pairs $x_{t_{2}}, x_{t} \in \mathbb{Z}^{d}$ satisfying $\left|x_{t_{2}}-\gamma(0)\right| \leq R t$ and $\left|x_{t}-\gamma(0)\right| \leq R t$ and $\left|x_{t_{2}}-x_{t}\right| \geq t^{1 / 4}$. Therefore, we conclude that

$$
\mathbb{P}\left(\mathcal{I}_{j}^{+} \cap G\right) \leq O\left(t^{2 d}\right)\left(1-\alpha^{C_{3}}\right)^{C_{4} t^{1 / 4}} .
$$

The last inequality immediately implies the lemma.

\section{Proof of Lemma 3.8}

Because $\omega \mapsto \tau_{h\left(\omega_{1}\right)} \omega$ is measure preserving on $\Omega$, we have

$$
\tilde{\mathbb{P}}\left(\left\{\left(\omega, \omega_{1}\right) \mid\left(\sigma_{j} \tilde{u}-\tilde{u}\right)^{2} 1_{\tilde{G}^{C}}>C_{0} t^{1 / 2}\right\}\right)=\mathbb{P}\left(\left\{\omega \mid\left(\sigma_{j} u-u\right)^{2} 1_{G^{C}}>C_{0} t^{1 / 2}\right\}\right)
$$

where the event $G \subset \Omega$ is defined by (4.46). So, on the event $G^{C}$ we know there is $\gamma \in M_{\delta}(\omega)$ such that

$$
|\gamma(t)-\gamma(0)|<t^{1 / 4}
$$

Let $B_{r}(x)$ denote the ball of radius $r>0$ centered at $x$. We may assume that there are at least two indices $j, k \in \mathbb{Z}^{d} \cap B_{t^{1 / 4}}(0)$ such that $\omega_{j}=a$ and $\omega_{k}=a$. This is because the event that $\omega_{\ell}=a$ for at most one of the cubes contained in $B_{t^{1 / 4}}(0)$ has probability less than $O\left(\beta^{N_{t}}\right)$ where $N_{t} \geq C t^{1 / 4}$ is the number of cubes contained in $B_{t^{1 / 4}}(0)$.

Let $\gamma \in M_{\delta}(\omega)$ with $|\gamma(t)-\gamma(0)| \leq t^{1 / 4}$. Then

$$
u(t, \omega) \leq g(\gamma(t))-a t+\delta .
$$

Suppose $\omega_{k}=a$ for some $k \neq j$ and $k \in B_{t^{1 / 4}}(0)$. Let $x_{k} \in Q_{k}$, so that $V\left(x_{k}, \omega\right)=a$. Define the path $\hat{\gamma}$ by

$$
\hat{\gamma}(s)=\left\{\begin{array}{cc}
\gamma(0)+s \frac{x_{k}-\gamma(0)}{t^{1 / 4}}, & s \in\left[0, t^{1 / 4}\right] \\
x_{k}, & s \in\left[t^{1 / 4}, t-t^{1 / 4}\right] \\
x_{k}+\left(s-t+t^{1 / 4}\right) \frac{\gamma(t)-x_{k}}{t^{1 / 4}}, & s \in\left[t-t^{1 / 4}, t\right] .
\end{array}\right.
$$

Then

$$
\sigma_{j} u(t, \omega) \geq g(\hat{\gamma}(t))-\mathcal{L}(\hat{\gamma}, \omega) \geq g(\gamma(t))-a\left(t-2 t^{1 / 4}-b 2 t^{1 / 4}-2 t^{1 / 4} \max _{|z| \leq 1} K(z) .\right.
$$

Therefore,

$$
u(t, \omega)-\sigma_{j} u(t, \omega) \leq(a-b) 2 t^{1 / 4}+2 t^{1 / 4} \max _{|z| \leq 1} K(z) .
$$

Hence $\left(u-\sigma_{j} u\right)^{2} \leq C_{0} t^{1 / 2}$ except possibly on a set of probability less than $O\left(\beta^{N_{t}}\right)$.

\section{Proof of Lemma 3.9 for $d=2$}

Assuming the non-degeneracy condition (1.6), we may choose real numbers $\nu>1$ and $\varepsilon_{0}>0$ such that $K(q) \geq|q|^{\nu}$ for all $q$ that satisfy $|q|<\varepsilon_{0}$. Having fixed $\zeta \in\left(\frac{\nu-1}{2 \nu-1}, \frac{1}{2}\right)$, we see that $\frac{\nu-1+\zeta}{\zeta \nu}<2$. So, we may choose $p \in\left(\frac{\nu-1+\zeta}{\zeta \nu}, 2\right)$.

Arguing by contradiction, we assume that $\# \Lambda_{j}>m^{p}$ : there are more than $m^{p}$ important cubes within the box $B_{j}=j-[0, m-1]^{d}$. Fix $\delta>0$ small. Consider a path $\gamma \in M_{\delta}(n, \omega)$. Let $\left[t_{1}, t_{2}\right]$ be 
the smallest interval containing all $s$ for which $\gamma(s) \in B_{j}$. Hence $\left|t_{2}-t_{1}\right| \geq C m^{p}$. Choose any one of the important cubes in $B_{j}$ and let $x_{c}$ denote its center point. Let us define a modified path $\hat{\gamma}$ as follows:

(i) For $s \in\left[0, t_{1}\right] \cup\left[t_{2}, t\right]$, let $\hat{\gamma}(s)=\gamma(s)$.

(ii) For $s \in\left[t_{1}, t_{2}\right]$ let

$$
\hat{\gamma}(s)= \begin{cases}\gamma\left(t_{1}\right)+\left(s-t_{1}\right) \cdot \frac{x_{c}-\gamma\left(t_{1}\right)}{m}, & s \in\left[t_{1}, t_{1}+m\right], \\ x_{c}, & s \in\left[t_{1}+m, t_{2}-m\right], \\ x_{c}+\left(s-t_{2}+m\right) \cdot \frac{\gamma\left(t_{2}\right)-x_{c}}{m}, & s \in\left[t_{2}-m, t_{2}\right] .\end{cases}
$$

We have the bound

$$
\mathcal{L}(\gamma)-\mathcal{L}(\hat{\gamma}) \geq-2 m \cdot \max _{|q| \leq 1} K(q)-2 m(b-a)+\int_{t_{1}}^{t_{2}} K\left(\gamma^{\prime}(s)\right) d s .
$$

We will prove that for sufficiently large $m$ the right side of (4.50) is larger than $\delta>0$, contradicting the fact that $\gamma \in M_{\delta}(n, \omega)$.

Let us denote by $J_{0} \subset\left[t_{1}, t_{2}\right]$ the set of times for which $\left|\gamma^{\prime}(s)\right| \geq \varepsilon_{0}$. Therefore, we may assume

$$
\int_{J_{0}} K\left(\gamma^{\prime}(s)\right) d s \leq 2 m\left(\max _{|q| \leq 1} K(q)+(b-a)\right)+\delta
$$

for otherwise the right side of (4.50) would be larger than $\delta$. Let $J_{1}=\left[t_{1}, t_{2}\right] \backslash J_{0}$; for these times $s \in J_{1}$ we have $\left|\gamma^{\prime}(s)\right| \leq \epsilon_{0}$. From (4.50) we also obtain:

$$
\begin{aligned}
\mathcal{L}(\gamma)-\mathcal{L}(\hat{\gamma}) & \geq-2 m\left(\max _{|q| \leq 1} K(q)+b-a\right)+\int_{J_{1}} K\left(\gamma^{\prime}(r)\right) d r \\
& \geq-2 m\left(\max _{|q| \leq 1} K(q)+b-a\right)+\int_{J_{1}}\left|\gamma^{\prime}(r)\right|^{\nu} d r \\
& \geq-2 m\left(\max _{|q| \leq 1} K(q)+b-a\right)+\left|J_{1}\right| \cdot\left(\frac{1}{\left|J_{1}\right|} \int_{J_{1}}\left|\gamma^{\prime}(r)\right| d r\right)^{\nu} .
\end{aligned}
$$

In the last line we applied Jensen's inequality. We will now prove that there exists a real number $\epsilon_{1}>0$ such that

$$
\int_{J_{1}}\left|\gamma^{\prime}(r)\right| d r \geq \epsilon_{1} m^{p}
$$

By our assumption, the number of important cubes within $B_{j}$ is more than $m^{p}$. Let us now paint all these cubes in $2^{d}$ colors so that no two cubes share the same color. By the pigeon-hole principle there are at least $m^{p} 2^{-d}$ important cubes having the same color. The distance between two cubes of the same color is at least 1 , hence we have $\int_{t_{1}}^{t_{2}}\left|\gamma^{\prime}(r)\right| d r \geq m^{p} 2^{-d}$. Therefore, since there is $C>0$ such that $\left|\gamma^{\prime}(s)\right| \leq C K\left(\gamma^{\prime}(s)\right)$ for all $s \in J_{0}$, we have

$$
\begin{aligned}
m^{p} 2^{-d} & \leq \int_{J_{1}}\left|\gamma^{\prime}(r)\right| d r+\int_{J_{0}}\left|\gamma^{\prime}(r)\right| d r \\
& \leq \int_{J_{1}}\left|\gamma^{\prime}(r)\right| d r+C \int_{J_{0}} K\left(\gamma^{\prime}(r)\right) d r \\
& \leq \int_{J_{1}}\left|\gamma^{\prime}(r)\right| d r+C(m+\delta) .
\end{aligned}
$$


In the last step we have applied (4.51). This last inequality implies (4.53), since $p>1$.

Now the inequalities (4.52) and (4.53) imply:

$$
\begin{aligned}
\mathcal{L}(\gamma)-\mathcal{L}(\hat{\gamma}) & \geq-2 m\left(\max _{|q| \leq 1} K(q)+b-a\right)+\left|J_{1}\right| \cdot\left(\frac{\epsilon_{1} m^{p}}{\left|J_{1}\right|}\right)^{\nu} \\
& =-2 m\left(\max _{|q| \leq 1} K(q)+b-a\right)+\left(\epsilon_{1}\right)^{\nu} m^{p \nu} \cdot\left|J_{1}\right|^{1-\nu} \\
& \geq-2 m\left(\max _{|q| \leq 1} K(q)+b-a\right)+\left(\epsilon_{1}\right)^{\nu} m^{p \nu} \cdot\left(m^{1 / \zeta}\right)^{1-\nu}
\end{aligned}
$$

In the last inequality we have used $\left|J_{1}\right| \leq n=m^{1 / \zeta}$. If we have

$$
p>\frac{\nu+\zeta-1}{\zeta \nu}
$$

then $p \nu+(1-\nu) / \zeta>1$. In this case, the right side of (4.55) is positive, and larger than $\delta$, for $t$ sufficiently large. Since this contradicts the approximate optimality of $\gamma \in M_{\delta}$, we must have $\# \Lambda_{j} \leq m^{p}$.

\section{Appendix}

Proof of Lemma 3.6: The bounds in (3.35) follow from the fact that $\mathbb{P}$ is the product measure on $\Omega=\{a, b\}^{\mathbb{Z}_{n}^{d}}$, with $\mathbb{P}(\omega(j)=a)=\alpha$ and $\mathbb{P}(\omega(j)=b)=\beta$. For every nonnegative integrable $\psi$, (3.35) implies

$$
\mathbb{E}(\psi)=\int \psi d \mathbb{P} \leq C^{\prime \prime} \int \psi d \mathbb{P} \circ \phi_{j}=\int \psi\left(\phi_{j} \omega\right) d \mathbb{P}=\mathbb{E}\left(\psi \circ \phi_{j}\right) .
$$

Similarly $\mathbb{E}(\psi) \geq C^{\prime} \mathbb{E}\left(\psi \circ \phi_{j}\right)$ for all such $\psi$.

Proof of Theorem 1.2; Let us define

$$
\Delta_{j} f(\omega)= \begin{cases}\beta\left(f\left(\phi_{j} \omega\right)-f(\omega)\right), & \text { if } \omega_{j}=a \\ \alpha\left(f\left(\phi_{j} \omega\right)-f(\omega)\right), & \text { if } \omega_{j}=b .\end{cases}
$$

Then Theorem 1.2 is a slight modification of the following

Theorem 5.1 ([14], Theorem 1.5) There is a constant $C>0$, such that

$$
\operatorname{var}(f) \leq C \cdot \sum_{j \in J} \frac{\left\|\Delta_{j} f\right\|_{2}^{2}}{1+\log \frac{\left\|\Delta_{j} f\right\|_{2}}{\left\|\Delta_{j} f\right\|_{1}}} .
$$

holds for all $f \in L^{2}\left(\Omega_{J}\right)$.

To derive Theorem 1.2 from this, we start with elementary observation

$$
C^{\prime}\left|\rho_{j} f(\omega)\right| \leq\left|\Delta_{j} f(\omega)\right| \leq C^{\prime \prime}\left|\rho_{j} f(\omega)\right|
$$

for $C^{\prime}=\min \{2 \alpha, 2 \beta\}$ and $C^{\prime \prime}=\max \{2 \alpha, 2 \beta\}$. Let $\kappa=\log \left(C^{\prime \prime} / C^{\prime}\right) \geq 0$. If $\log \frac{\left\|\rho_{j} f\right\|_{2}}{\left\|\rho_{j} f\right\|_{1}} \geq 2 \kappa$, then

$$
\log \frac{\left\|\Delta_{j} f\right\|_{2}}{\left\|\Delta_{j} f\right\|_{1}} \geq \log \frac{C^{\prime}}{C^{\prime \prime}}+\log \frac{\left\|\rho_{j} f\right\|_{2}}{\left\|\rho_{j} f\right\|_{1}} \geq \frac{1}{2} \log \frac{\left\|\rho_{j} f\right\|_{2}}{\left\|\rho_{j} f\right\|_{1}} .
$$


Consequently, Theorem 5.1 implies

$$
\operatorname{var}(f) \leq C \cdot \sum_{j \in J} \frac{\left\|\Delta_{j} f\right\|_{2}^{2}}{1+\log \frac{\left\|\Delta_{j} f\right\|_{2}}{\left\|\Delta_{j} f\right\|_{1}}} \leq 2\left(C^{\prime \prime}\right)^{2} C \sum_{j \in J} \frac{\left\|\rho_{j} f\right\|_{2}^{2}}{1+\log \frac{\left\|\rho_{j} f\right\|_{2}}{\left\|\rho_{j} f\right\|_{1}}} .
$$

On the other hand, if $\log \frac{\left\|\rho_{j} f\right\|_{2}}{\left\|\rho_{j} f\right\|_{1}} \in[0,2 \kappa)$, then Theorem 5.1 implies

$$
\operatorname{var}(f) \leq C \cdot \sum_{j \in J}\left\|\Delta_{j} f\right\|_{2}^{2} \leq(1+2 \kappa) 2\left(C^{\prime \prime}\right)^{2} C \sum_{j \in J} \frac{\left\|\rho_{j} f\right\|_{2}^{2}}{1+\log \frac{\left\|\rho_{j} f\right\|_{2}}{\left\|\rho_{j} f\right\|_{1}}}
$$

\section{References}

[1] S. Armstrong, P. Cardaliaguet, P.E. Souganidis, Error estimates and convergence rates for the stochastic homogenization of Hamilton-Jacobi equations, preprint 2012, arXiv:1206.2601.

[2] S. Armstrong and P.E. Souganidis, Stochastic homogenization of level-set convex HamiltonJacobi equations, preprint 2012, arXiv:1203.6303.

[3] M. Bardi and I. Capuzzo-Dolcetta, Optimal Control and Viscosity Solutions of Hamilton-JacobiBellman Equations, Birkhäuser, 1997.

[4] I. Benjamini, G. Kalai; O. Schramm, First passage percolation has sublinear distance variance, Ann. Probab. 31 (2003), pp. 1970-1978.

[5] M. Benaïm and R. Rossignol, Exponential concentration for first passage percolation throuh modified Poincaré inequalities, Ann. Inst. Henri Poincaré - Prob. Stat. 44 (2008), pp. 544-573.

[6] E. Kosygina and S.R.S. Varadhan, Homogenization of Hamilton-Jacobi-Bellman equations with respect to time-space shifts in a stationary ergodic medium, Comm. Pure Appl. Math, 61 (2008) pp. 816-847.

[7] E. Kosygina, F. Rezakhanlou, S.R.S. Varadhan, Stochastic homogenization of Hamilton-JacobiBellman equations, Comm. Pure Appl. Math 59 (2006), pp. 1489-1521.

[8] P.-L. Lions and P.E. Souganidis, Homogenization of "viscous" Hamilton-Jacobi equations in stationary ergodic media, Comm. PDE 30 (2005), pp. 335375.

[9] P.-L. Lions and P.E. Souganidis, Stochastic homogenization of Hamilton-Jacobi and "viscous"Hamilton-Jacobi equations with convex nonlinearities- revisited, Comm. Math. Sci. 8 (2010). pp. 672-637.

[10] F. Rezakhanlou, Central limit theorem for stochastic Hamilton-Jacobi equations, Comm. Math. Phys. 211 (2000), pp. 413-438.

[11] F. Rezakhanlou and J. E. Tarver. Homogenization for stochastic Hamilton-Jacobi equations, Arch. Ration. Mech. Anal., 151 (2000), pp. 277-309.

[12] R. Schwab, Stochastic homogenization of Hamilton-Jacobi equations in stationary ergodic spatiotemporal media, Indiana Univ. Math. J. 58 (2009), pp. 527-581. 
[13] P.E. Souganidis, Stochastic homogenization of Hamilton-Jacobi equations and some applications, Asymptotic Analysis, 20 (1999), pp. 1-11.

[14] M. Talagrand, On Russo's approximate zero-one law., Ann. of Probab. 22 (1994), pp. 1576-1587. 